\title{
Involvement of matrix metalloproteinase-2 in the development of renal interstitial fibrosis in mouse obstructive nephropathy
}

\author{
Xuanyi Du²,3, Akira Shimizu', Yukinari Masuda ${ }^{1}$, Naomi Kuwahara', Takashi Arai ${ }^{1}$, Mitue Kataoka', \\ Masaaki Uchiyama', Tomohiro Kaneko², Toshio Akimoto ${ }^{4}$, Yasuhiko lino ${ }^{2}$ and Yuh Fukuda ${ }^{1}$
}

Renal fibrosis is a common finding in progressive renal diseases. Matrix metalloproteinases (MMPs) are involved in epithelial-to-mesenchymal transition (EMT). We investigated the role of MMP-2 and the effect of inhibition of MMPs on the development of renal fibrosis. Renal fibrosis was induced in MMP-2 wild-type (MMP- $2^{+/+}$) mice by unilateral ureteral obstruction (UUO). Renal histopathology, EMT-associated molecules, and activity of MMP-2 and MMP-9 were examined during the development of interstitial fibrosis. UUO-renal fibrosis was also induced in MMP-2 deficient $\left(\mathrm{MMP}_{-} 2^{-/-}\right.$) and MMP-2 $2^{+/+}$mice treated with minocycline (inhibitor of MMPs). In MMP-2 $2^{+/+}$mice, MMP-2 and MMP-9 were expressed in damaged tubules, and their activities increased in a time-dependent manner after UUO. Interstitial fibrosis was noted at day 14, with deposition of types III and I collagens and expression of markers of mesenchymal cells (S100A4, vimentin, $\alpha$-smooth muscle actin, and heat shock protein-47) in damaged tubular epithelial cells, together with F4/80 + macrophage infiltration. Fibrotic kidneys expressed EMT-associated molecules (ILK, TGF- $\beta 1$, Smad, Wnt, $\beta$-catenin, and Snail). In contrast, the kidneys of MMP- $2^{-1-}$ mice and minocycline-treated MMP-2 ${ }^{+/+}$mice showed amelioration of renal fibrosis with reduced expression of markers of mesenchymal cells in tubular epithelial cells, inhibition of upregulated EMT-associated molecules, and suppression of macrophage infiltration. The results suggested that MMP-2 have a pathogenic role in renal interstitial fibrosis, possibly through the induction of EMT and macrophage infiltration. Inhibition of MMPs may be beneficial therapeutically in renal fibrosis.

Laboratory Investigation (2012) 92, 1149-1160; doi:10.1038/labinvest.2012.68; published online 21 May 2012

KEYWORDS: epithelial-to-mesenchymal transition; MMP-2; MMP inhibitor; renal fibrosis; tubular epithelial cell

Renal interstitial fibrosis is considered the final common pathway of progressive kidney disease leading to end-stage renal failure. ${ }^{1-3}$ It is characterized histopathologically by loss of renal tubules, increased number of myofibroblasts, and accumulation of extracellular matrix (ECM). ${ }^{4-6}$ Recent studies have emphasized the potential role of epithelial-tomesenchymal transition (EMT) in the development of renal interstitial fibrosis. ${ }^{7-12}$ Tubular epithelial cells can acquire a mesenchymal phenotype after several injuries, enhance migratory capacity enabling them to transit from the renal tubular microenvironment into the interstitial space, and contribute to the development of renal fibrosis as ECMproducing myofibroblasts. The role of EMT in renal fibrogenesis has been suggested in several studies of cultured cells, animal models of kidney diseases, and human nephropathies and kidney transplantation, ${ }^{7-12}$ although several recent studies also demonstrate the doubt on this mechanism as a major contributor to renal fibrosis. ${ }^{13-15}$

Matrix metalloproteinases (MMPs) are a family of zincdependent proteases responsible for ECM turnover as well as degradation of bioactive proteins. ${ }^{16-18}$ This family includes collagenases, gelatinases, stromelysins, and membrane-type MMPs. In the EMT sites during renal fibrogenesis, MMPs mediate disruption of the integrity of the tubular basement membrane (TBM), which in turn promotes tubular epithelial cell EMT, and progressive interstitial fibrosis. ${ }^{19-21}$ Recent

\footnotetext{
${ }^{1}$ Department of Pathology (Analytic Human Pathology), Nippon Medical School, Tokyo, Japan; ${ }^{2}$ Department of Internal Medicine (Neurology, Nephrology and Rheumatology), Nippon Medical School, Tokyo, Japan; ${ }^{3}$ Department of Nephrology, Second Affiliated Hospital of Harbin Medical University, Harbin, Heilongjiang, China and ${ }^{4}$ Division of Laboratory Animal Science, Nippon Medical School, Tokyo, Japan

Correspondence: Dr A Shimizu, MD, PhD, Department of Pathology (Analytic Human Pathology), Nippon Medical School, 1-1-5, Sendagi, Bunkyo-ku, Tokyo 113-8602, Japan.

E-mail: ashimizu@nms.ac.jp
}

Received 26 October 2011; revised 23 February 2012; accepted 10 March 2012 
studies have demonstrated that MMPs, especially gelatinases (MMP-2 and MMP-9) have major roles in the EMT process, and are involved in the renal tubular cell EMT both in vivo and in vitro. Although specific therapies that inhibit the progression of chronic renal disease are currently not available, modulation of MMPs in the EMT seems a legitimate therapeutic target with the aim of inhibition of renal fibrogenesis. With regard to MMP-9, a recent study demonstrated that mice lacking the MMP-9 gene show mild renal interstitial fibrosis in unilateral ureteral obstruction (UUO). ${ }^{22}$ However, the therapeutic effect of MMP-2 inhibition on progressive renal fibrosis is still uncertain.

This study was designed to determine the involvement of MMP-2 during the development of renal fibrosis in a UUO model. UUO is a well-established experimental model of renal injury leading to progressive interstitial fibrosis. ${ }^{23,24}$ After demonstrating the activation of MMP-2, experiments were performed to determine the therapeutic effects of MMP inhibition on progressive renal fibrosis, in both MMP-2 knockout (MMP- $2^{-1-}$ ) mice and mice treated with minocycline, a MMP inhibitor.

\section{MATERIALS AND METHODS \\ Mice}

MMP- $2^{-/-}$mice generated in C57BL/6 genetic background by gene targeting were kindly provided by Shigeyoshi Itohara (Laboratory for Behavioral Genetics, RIKEN Brain Science Institute, Saitama, Japan). ${ }^{25}$ All experimental procedures described here were approved by the Nippon Medical School Animal Studies Committee.

\section{Renal UUO Model}

Three experiments were performed in this study using 12-week-old male MMP-2 wild-type (MMP-2 ${ }^{+/+}$) and MMP- $2^{-/-}$mice. In experiment 1 , renal interstitial fibrosis was induced in MMP- $2^{+/+}$mice by UUO operation, using the same procedures described previously. ${ }^{23,24}$ After the induction of general anesthesia by intraperitoneal (IP) injection of pentobarbital $(40-70 \mathrm{mg} / \mathrm{kg}$ body wt), the mice were subjected to either UUO or sham operation. In UUO animals, the lower $1 / 3$ portion of left ureter between the kidney and the bladder was identified through a small suprapubic incision and was ligated with 4-0 silk at two points and cut between the ligatures in order to prevent retrograde urinary tract infection. The sham operation consisted of a similar suprapubic incision and identification of the left ureter, but no ligation of the ureter was performed. These animals were used to obtain control kidneys. After UUO/sham operation, the mice ( $n=5$ at each time point) were killed at $3,5,7,10$, and 14 days after operation. Experiment 2 involved exactly the same UUO or sham operation but was performed in MMP- $2^{-l-}$ mice. The mice ( $n=5$ at each time point) were killed at $3,5,7,10$, and 14 days after operation. In experiment 3, MMP- $2^{+1+}$ mice with UUO were treated with minocycline (Sigma, St Louis, MO, USA) as MMP-2 and
MMP-9 inhibitor, from $36 \mathrm{~h}$ before to 14 days post-UUO. Minocycline $45 \mathrm{mg} / \mathrm{kg}$ in dimethyl sulfoxide (DMSO) and $0.9 \% \mathrm{NaCl}$ or an equal volume of DMSO and $0.9 \% \mathrm{NaCl}$ (placebo) was injected IP at $36 \mathrm{~h}$ before renal UUO and was followed by $22.5 \mathrm{mg} / \mathrm{kg}$ minocycline or placebo IP every $12 \mathrm{~h}$ for 14 days post-UUO, based on the protocol described previously. ${ }^{26}$ Mice ( $n=5$ at each time point) treated with minocycline were killed at days 5 and 14 .

\section{Histopathological, Immunohistochemical, and Electron Microscopic Examinations}

The left kidneys harvested from the UUO and sham-operated animals were fixed in $20 \%$ buffered formalin and embedded in paraffin for light microscopy. Tissues were stained with hematoxylin and eosin, periodic acid-Schiff, Masson trichrome (Masson), and periodic acid-methenamine Silver for histopathological examination.

The following primary antibodies were used for immunohistochemistry: (a) goat polyclonal anti-MMP-2 (Santa Cruz Biotechnology, Santa Cruz, CA, USA) to detect MMP-2-producing cells, (b) goat polyclonal anti-MMP-9 (Santa Cruz Biotechnology) to detect MMP-9-producing cells, (c) rabbit polyclonal anti-S100A4 (Thermo Scientific, Middletown, VA, USA), also known as fibroblast-specific protein 1 and thought to be a marker of the mesenchymal cells, (d) mouse monoclonal anti-vimentin (M851; Dako, Glostrup, Denmark), an intermediate filament protein expressed only in mesenchymal cells, (e) mouse monoclonal anti- $\alpha$-smooth muscle actin ( $\alpha \mathrm{SMA})$, a marker for myofibroblasts, (f) mouse monoclonal anti-heat shock protein-47 (HSP-47, Stressgen, Victoria, BC, Canada), a collagen-binding stress protein and thought to be a collagen-specific molecular chaperone and a marker of collagen synthesis, (g) goat polyclonal anti-type III collagen (Southern Biotechnology, Birmingham, AL, USA), to evaluate the deposition of type III collagen, and (h) goat polyclonal anti-type I collagen (Southern Biotechnology), to evaluate the deposition of type I collagen, and (i) rat monoclonal anti-mouse F4/80 (AbDserotec, Oxford, UK), to evaluate the macrophage infiltration.

Immunohistochemistry for MMP-2 and MMP-9 as well as two-color immunohistochemistry for $\alpha$ SMA (Texas Red) and S100A4 (FITC) were performed using frozen tissue sections and standard indirect technique, followed by examination under a fluorescence microscope. For immunohistochemistry for S100A4, vimentin, $\alpha$ SMA, HSP-47, type III collagen, and type I collagen, formalin-fixed and paraffin-embedded tissue sections were used and the specimens were stained by the standard avidin-biotin-peroxidase complex technique. In order to optimize the detection of S100A4 and vimentin, sections were microwaved for $10 \mathrm{~min}$ in $0.01 \mathrm{M}$ sodium citrate ( $\mathrm{pH}$ 6.0) before incubation with the primary antibody. To detect HSP-47, type III collagen, and type I collagen, sections were incubated with $0.1 \%$ pepsin for 15 to $30 \mathrm{~min}$ before incubation with primary antibody. 
For electron microscopic examination, the left kidney tissue was fixed in $2.5 \%$ glutaraldehyde solution in phosphate buffer ( $\mathrm{pH}$ 7.4) and postfixed with $1 \%$ osmium tetroxide, dehydrated, and embedded in Epok 812. Ultrathin sections were stained with uranyl acetate and lead citrate, and then examined with an electron microscope (model H7100, Hitachi, Tokyo, Japan).

The area of Masson stain + fibrosis and the expression of type III and type I collagens, S100A4, vimentin, $\alpha \mathrm{SMA}$, and HSP-47 was analyzed quantitatively using a computerassisted image analysis system comprising an Olympus BX60 microscope connected via a video camera to a PC. Data were analyzed using the WinROOF image processing software (Mitani, Tokyo, Japan). At least 20 digitized images of the renal cortex taken at $\times 200$ magnification $\left(0.569 \mathrm{~mm}^{2}\right)$ were analyzed in each sample, and the percentage area of positive staining per field was evaluated.

\section{Gelatin and In Situ Zymography}

Supernatant of $10 \mu \mathrm{g}$ of total protein from the homogenate of the left kidney harvested from each animal was used for gelatin zymography, as described previously. ${ }^{26}$ Briefly, electrophoresis was carried out on $10 \%$ polyacrylamide gels containing $0.1 \%$ gelatin under non-reducing conditions. After electrophoresis, the gels were washed in $2.5 \%$ Triton
$\mathrm{X}-100$ to remove sodium dodecyl sulfate, incubated for $16 \mathrm{~h}$ at $37^{\circ} \mathrm{C}$, and stained with $0.1 \%$ Coomassie Brilliant Blue R250 (Sigma). To test whether minocycline inhibits MMP-2 and MMP-9 activities, minocycline $(0.5$ or $10 \mathrm{mg} / \mathrm{ml})$ was added to the rinse and reaction buffers during the development of the zymograms. MMP-2 and MMP-9 activities were estimated from their gelatinolytic activities. Densitometric analysis of the gels was performed using NIH image software (Image, v.1.62; National Institutes of Health, Bethesda, MD, USA). For mapping of gelatinolytic activity in the injured left kidney, in situ zymography was performed using the method described previously. ${ }^{27}$ Gelatin-FITC films was prepared as described previously, ${ }^{27}$ and cryostat-cut kidney sections (4-6 mm thickness) were placed on the film, and incubated at $37^{\circ} \mathrm{C}$ for 6 to $32 \mathrm{~h}$. After incubation, the films were examined under immunofluorescent microscope.

\section{Real-Time PCR for EMT-Related Molecules}

To examine the mRNA levels of the interstitial-type collagen, type III and type I collagens, the gelatinase-related molecules, MMP-2, MMP-9, TIMP-1, and TIMP-2, and the EMT-related molecules, integrin-linked kinase (ILK), transforming growth factor- $\beta 1$ (TGF- $\beta 1$ ), Smad 2 , Wnt, $\beta$-catenin, Snail 1 , and Snail 2, real-time PCR was performed using renal cortex tissues. The total RNA was extracted from the renal cortex by
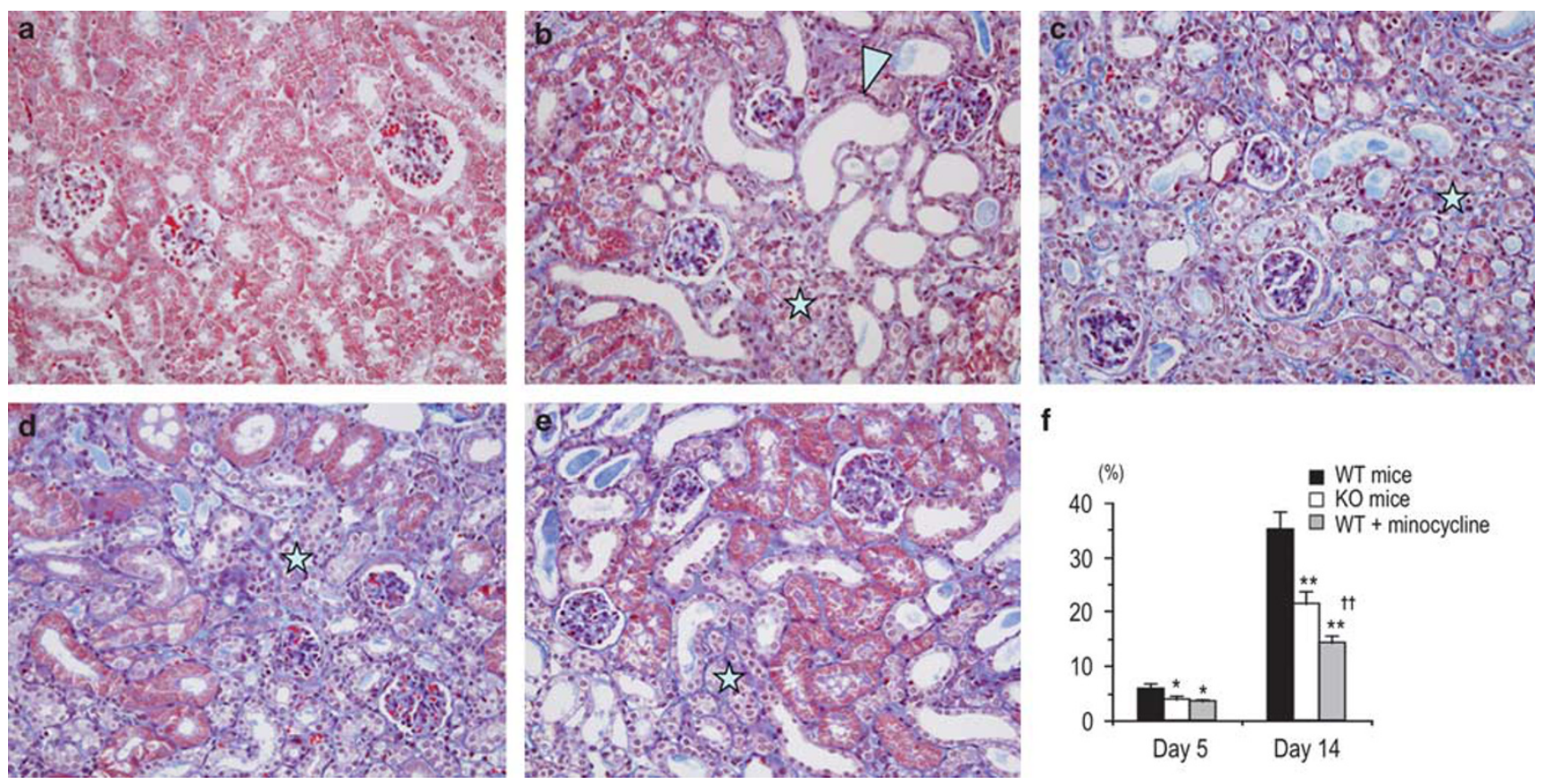

Figure 1 Renal fibrosis in MMP-2 $2^{+/+}$mice, MMP- $2^{-/-}$mice, and MMP-2 $2^{+/+}$mice treated with minocycline after unilateral ureteral obstruction (UUO) $(\mathbf{a}-\mathbf{e}$ : Masson stain, $\times 200)$. Under normal conditions $(\mathbf{a})$, note the lack of damaged tubules and absence of interstitial expansion. In MMP- $2^{+/+}$mice at day 5 (b) and day 14 (c) post-UUO, atrophy (asterisk) or dilated (arrowhead) damaged tubules were noted with the development of interstitial fibrosis. In MMP- $2^{-/-}$mice (d) and MMP-2 $2^{+/+}$mice treated with minocycline (e) at day 14 post-UUO, the degree of interstitial fibrosis was milder than MMP-2 $2^{+/+}$ mice, although atrophic damaged tubules (asterisk) and expansion of interstitial fibrosis were seen. The percentage of Masson stain + fibrotic areas in renal cortex (f) showed that renal fibrosis was significantly diminished in MMP-2 $2^{-1-}$ mice (KO mice) and MMP-2 ${ }^{+/+}$mice treated with minocycline (WT + minocycline) , compared with MMP-2 ${ }^{+1+}$ mice (WT mice) at day 5 and day 14 post-UUO. Data are mean $\pm \mathrm{s}$.d. of $n=5$ mice. ${ }^{\star} P<0.05$ and ${ }^{* * P}<0.001$ are compared with MMP-2 ${ }^{+/+}$mice. ${ }^{\dagger \dagger} P<0.001$ is on MMP-2 ${ }^{+/+}$mice treated with minocycline, which is compared with MMP-2 ${ }^{-/-}$mice. 
Qiagen RNeasy Mini kit (Qiagen, Hilden, Germany). A cDNA copy was created with reverse transcriptase from High Capacity RT-kit (Applied Biosystems, Foster City, CA, USA). Gene expression was analyzed by real-time quantitative RTPCR using the TaqMan system based on real-time detection of accumulated fluorescence according to the manual supplied by the manufacturer (ABI PRISM- 7900HT; Applied Biosystems). The normalized value for mRNA expression in each sample was calculated as the relative quantity of relevant primers divided by the relative quantity of the housekeeping gene mouse GAPDH (4352339E). The sequences of the TaqMan sets were as follows: MMP-2 (Mm00439498_m1), MMP-9 (Mm00442991_m1), TIMP-1 (Mm00441818_m1), TIMP-2 (Mm00441825_m1), type I collagen, alpha 1 (Mm00801666_g1), type III collagen, alpha 1 (Mm01254476_ m1), ILK (Mm00439671_g1), TGF- $\beta$ (Mm03024053_m1), Smad 2 (Mm00487530_m1), Wnt (Mm01300555_g1), $\beta$-catenin (Mm00483039_m1), Snail 1 (Mm0041533_g1), and Snail 2 (Mm00441531_m1). Quantification was performed using SDS 2.1 software.

\section{Statistical Analysis}

All data were expressed as mean \pm s.d. When multiple groups were analyzed simultaneously, the group comparison of one-way ANOVA (analysis of variance) with Tukey's HSD post hoc was used. If a Welch-ANOVA was conducted, Games-Howell method was applied. When two groups were analyzed, the unpaired Student's $t$-test was employed. Statistical significance was set at $P<0.05$.
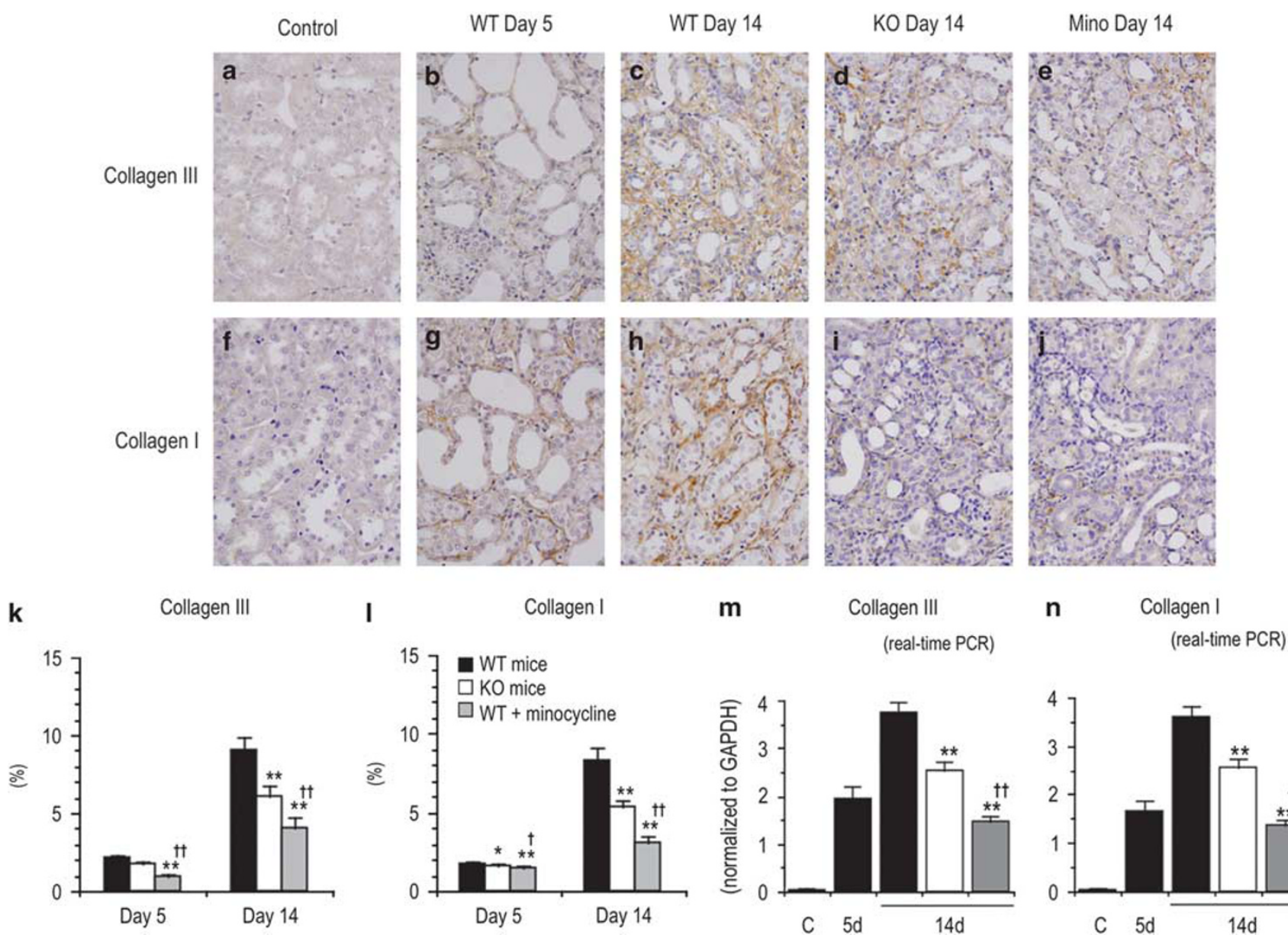

Figure 2 The deposition of collagen III $(\mathbf{a}-\mathbf{e}, \times 400)$ and collagen I $(\mathbf{f}-\mathbf{j}, \times 400)$ in MMP- $2^{+/+}$mice, MMP- $2^{-/-}$mice, and MMP-2 $2^{+/+}$mice treated with minocycline after UUO. Under normal conditions (Control), note the lack of increased deposition of type III (a) and type I (f) collagens. In MMP-2 ${ }^{+/+}$mice at day 5 (b, g: WT Day 5) and day 14 (c, h: WT Day 14) post-UUO, the deposition of type III (b, c) and type I (g, h) collagens developed. In MMP- ${ }^{-1-}$ mice (d, i: KO Day 14) and MMP-2 $2^{+/+}$mice treated with minocycline (e, j: Mino Day 14) at day 14 post-UUO, the deposition of type III (d, e) and type I (i, j) collagens was milder than MMP-2 $2^{+/+}$mice. Quantitative analysis of the percentage areas in cortex $(\mathbf{k}, \mathbf{I})$ and mRNA levels $(\mathbf{m}, \mathbf{n})$ of type III $(\mathbf{k}, \mathbf{m})$ and type I (I, $\left.\mathbf{n}\right)$ collagens showed that the production and the deposition of type III and type I collagens were significantly diminished in MMP-2 $2^{-I-}$ mice (KO mice) and MMP-2 $2^{+1+}$ mice treated with minocycline (WT + minocycline), compared with MMP- $2^{+/+}$mice (WT mice). Data are mean $\pm \mathrm{s}$.d. of $n=5$ mice. ${ }^{\star P}<0.05$ and ${ }^{* * P}<0.001$ are compared with MMP-2 ${ }^{+1+}$ mice. ${ }^{\dagger} P<0.05$ and ${ }^{\dagger} P<0.001$ are on MMP-2 ${ }^{+/+}$mice treated with minocycline, which is compared with MMP $-2^{-1-}$ mice. 


\section{RESULTS}

\section{UUO in MMP-2 $2^{+/}$Mice}

In MMP-2 ${ }^{+/+}$mice after UUO, renal tubular injury and interstitial fibrosis occurred by day 5 , and developed at day 14 (Figure 1). Interstitial fibrosis at day 14 was characterized by the expansion of Masson stain + fibrotic areas and the increased production and deposition of type III and type I collagens (Figures 1 and 2). Together with or before the development of interstitial fibrosis, extensive tubular injury was noted with epithelial cell damage and loss of epithelial cell polarity. Analysis of MMP-2 and MMP-9 activities in the renal cortex using gelatin zymography (Figure 3) showed that UUO caused increased MMP-2 and MMP-9 activities in MMP- $2^{+/+}$mice at least at day 3. Subsequently, the activities of both MMP-2 and MMP-9 increased in a timedependent manner and reached highest levels at day 14, during the progression of interstitial fibrosis.

We also examined the expression of MMP-2 and MMP-9 in renal tissues in MMP- $2^{+/+}$mice before UUO and at day 14 post-UUO (Figure 4 ), when MMP activity was the highest during the development of interstitial fibrosis, as demonstrated on gelatin zymography. At baseline, MMP-2 and
MMP-9 were not detected in the kidney. However, at day 14 post-UUO, the expression levels of MMP-2 and MMP-9 increased on damaged tubular epithelial cells in the dilated or atrophic damaged tubules.

In real-time PCR study, the production of MMP-2 and MMP-9 upregulated at day 14 post-UUO in MMP-2 $2^{+1+}$ mice, although very low levels of these were noted before UUO or sham control mice. In addition, similar tendency of TIMP-1 and TIMP-2 production was seen before UUO and at day 14 post-UUO in MMP-2 ${ }^{+/+}$mice. Examination of MMP-2 and MMP-9 activities by in situ zymography in tissue sections showed gelatinase activity mainly in the dilated or atrophic renal tubules at day 14 (Figure 5), consistent with the expression of MMP-2 and MMP-9 in immunofluorescent study.

During the development of interstitial fibrosis, damaged tubular epithelial cells and interstitial cells exhibited mesenchymal and collage-producing phenotypes (Figure 6). S100A $4+$, vimentin,$+ \alpha \mathrm{SMA}+$, and HSP-47 + tubular epithelial cells were occasionally present in damaged tubules (Figure 7). In addition, only a very small number, but both $\mathrm{S} 100 \mathrm{~A} 4+$ and $\alpha \mathrm{SMA}+$ myofibroblasts were noted in a

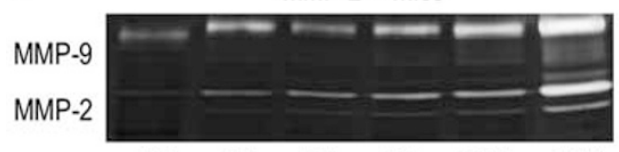

$\begin{array}{llllll}\text { D0 } & \text { D3 } & \text { D5 } & \text { D7 } & \text { D10 } & \text { D14 }\end{array}$

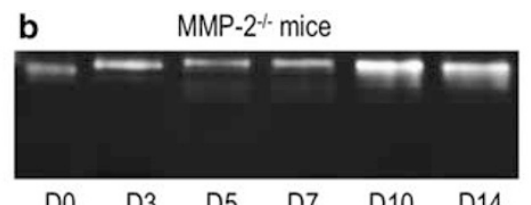

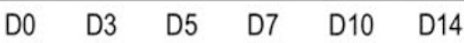

C MMP-9 activity

MMP-2 activity

d

MMP-9 activity
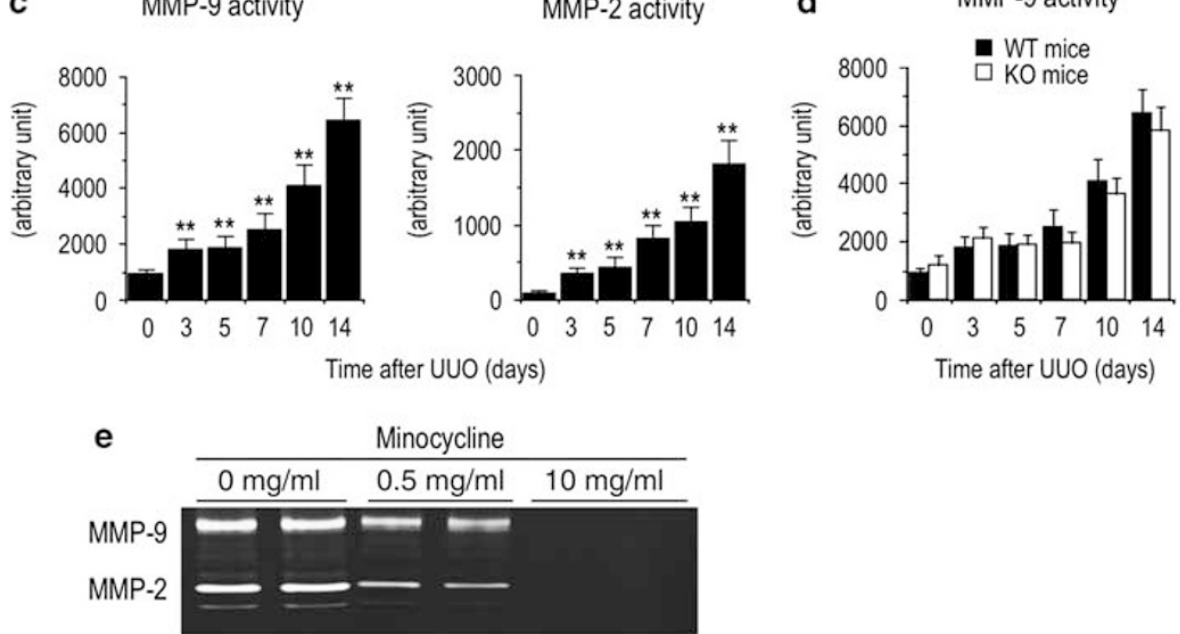

Figure 3 MMP-2 and MMP-9 activities examined by gelatin zymography. There are representative examples of gelatin zymography of five experiments during the development of renal fibrosis before (D0), and at day 3 (D3), day 5 (D5), day 7 (D7), day 10 (D10) and day 14 (D14) post-unilateral ureteral obstruction (UUO) in MMP- $2^{+1+}$ mice (a) and MMP- $2^{-1-}$ mice (b). These graphs are quantitative data of MMP- 2 and MMP-9 activities during the development of renal fibrosis in MMP- $2^{+1+}$ mice (c). Data are mean \pm s.d. of five animals. ${ }^{*} P<0.001$ is compared with day 0 (before UUO). This graph shows the comparison of MMP-9 activity at day 0 to day 14 between MMP- $2^{+/+}$mice (WT mice) and MMP- $2^{-/-}$mice (KO mice) (d). No significantly differences were evident between MMP-2 $2^{+1+}$ mice and MMP- $2^{-1-}$ mice. Data are mean \pm s.d. of five mice each. This is representative example of gelatin zymography incubated with different concentrations of minocycline $(0,0.5$, and $10 \mathrm{mg} / \mathrm{ml})(\mathbf{e})$. An equal aliquot of MMP proteins was added to each lane and gels were incubated with different concentrations of minocycline. Minocycline inhibited MMP-2 and MMP-9 activities, which is indicated by the decreased gelatinolytic bands, as dose-dependent manner. 

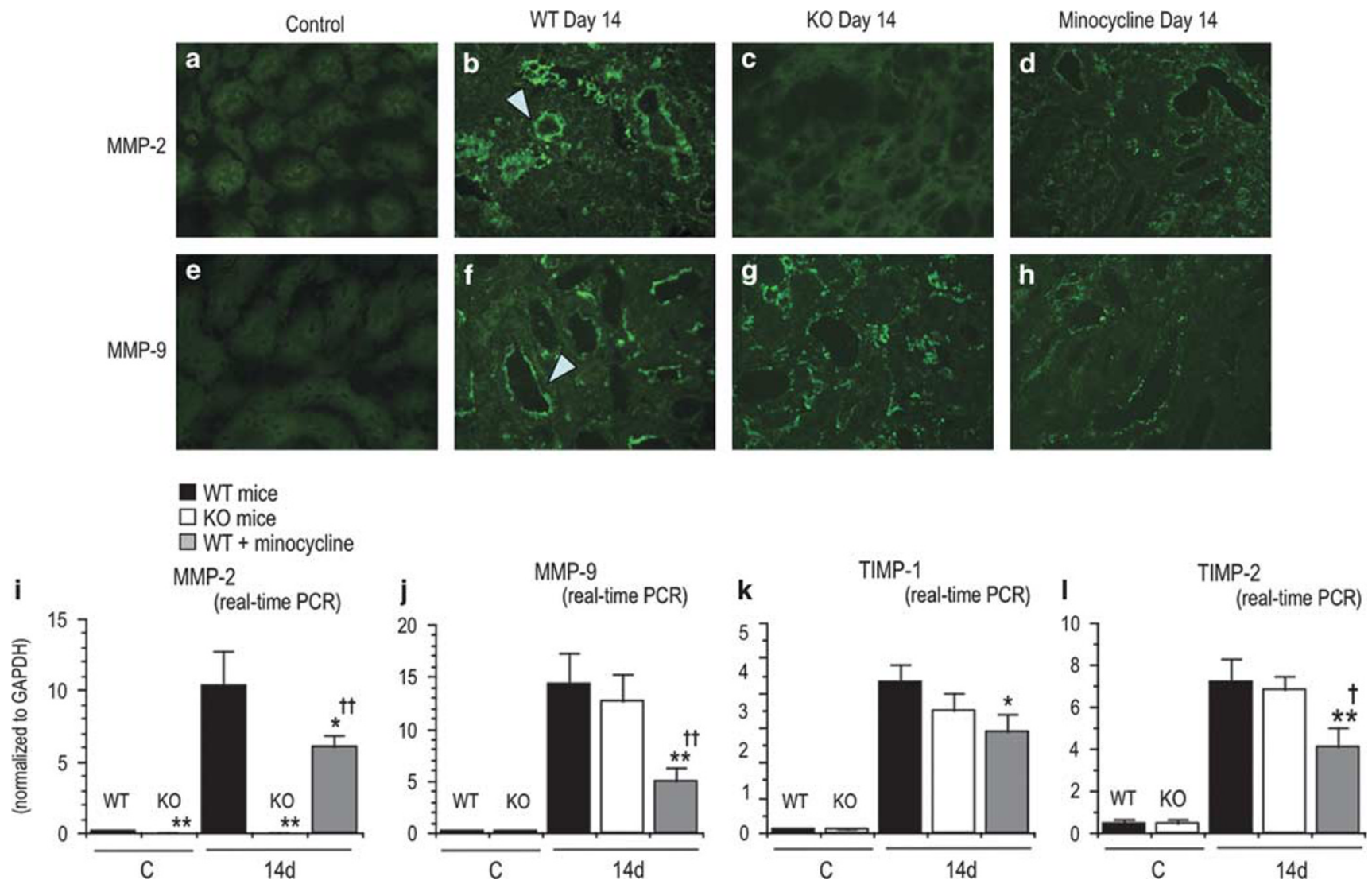

Figure 4 The expression of MMP-2 (a-d: $\times 400)$ and MMP-9 (e-h: $\times 400)$, and the mRNA levels of MMP-2 (i), MMP-9 (j), TIMP-1 (k), and TIMP-2 (I) in MMP$2^{+/+}$mice, MMP- $2^{-/-}$mice, and MMP- $2^{+/+}$mice treated with minocycline after unilateral ureteral obstruction (UUO). At baseline (Control), tubular epithelial cells showed no or minimal MMP-2 (a) and MMP-9 (e) expression. However, in MMP-2 ${ }^{+1+}$ mice at day 14 post-UUO (WT Day 14), tubular epithelial cells in dilated and atrophic tubules showed upregulation of MMP-2 (arrowhead in panel b) and MMP-9 (arrowhead in panel f). In MMP-2 $2^{-1}$ mice at day 14 (KO Day 14), although MMP-2 expression could not be detected (c), similar degree of MMP-9 was noted between MMP- $2^{-/-}$mice (g) and MMP-2 $2^{+/+}$mice (f). In MMP-2 $2^{+/+}$mice treated with minocycline at day 14 (minocycline Day 14), the expression of MMP-2 (d) and MMP-9 (h) decreased than MMP-2 ${ }^{+/+}$ mice $(\mathbf{b}, \mathbf{f})$. In real-time PCR studies, although only a few or no detectable levels of mRNA were noted in MMP-2 (i), MMP-9 (j), TIMP-1 (k), and TIMP-2 (I) before UUO (C: control) in both the MMP-2 ${ }^{+/+}$mice (WT) and MMP- $2^{-/-}$mice (KO), they were upregulated at day 14 post-UUO (14 d), except for MMP-2 in MMP-2 $2^{-1-}$ mice. Minocycline inhibited the upregulation of MMP-2, MMP-9, TIMP-1, and TIMP-2 at day 14 post-UUO (14 d). Data are mean \pm s.d. of five animals. ${ }^{*} P<0.05$ and ${ }^{* *} P<0.001$ are compared with MMP- $2^{+/+}$mice. ${ }^{\dagger} P<0.05$ and ${ }^{\dagger \dagger} P<0.001$ are on MMP- $2^{+/+}$mice treated with minocycline, which is compared with $\mathrm{MMP}^{-2^{-1-}}$ mice.

damaged tubules (Figure 7). Many F4/80 + macrophages also infiltrated the interstitium, mainly around the damaged tubules (Figure 6).

The development of renal interstitial fibrosis was coupled with the transition of damaged tubular epithelial cells to mesenchymal and collagen-producing phenotypes (Figure 8). In addition, many mesenchymal-like cells, probably myofibroblasts accumulated in interstitium. Upregulation of EMTrelated molecules such as intracellular signal transduction pathways, ILK, TGF- $\beta / \mathrm{Smad}$, and $\mathrm{Wnt} / \beta$-catenin signaling, and transcription factor Snail was noted at 5 and 14 days of renal fibrogenesis (Figure 9).

Electron microscopy at day 14 post-UUO in MMP-2 $2^{+/+}$ mice showed the loss of basolateralin folding and loss of surface microvilli in the damaged epithelial cells, indicating loss of cell polarity and loss of cell differentiation (Figure 10). Damaged tubular epithelial cells also contained filamentous actin bundles on the basal side of the epithelium, which is one of the findings of EMT. TBM in damaged atrophic tubules showed morphological changes of TBM, characterized by redundant, irregularly thickened, and tortuous profiles, indicating the remodeling of TBM. The contact between damaged tubular epithelial cells and damaged TBM became irregular. Spindleshape tubular epithelial cells were noted without obvious tight junctions. Fibroblast-like mesenchymal cells were observed in the basal area of the tubules between the damaged epithelial cells and TBM. The foci of EMT were characterized by TBM disruption and interstitial invasion of spindle-shape epithelial cells with accumulation of fibroblasts around the TBM. During the development of interstitial fibrosis, electron microscopy showed loss of polarity and differentiation in damaged epithelial cells, and migration of spindle-shape tubular epithelial 
cells into the interstitium through the ruptured TMB, indicating the development of EMT.

\section{UUO in MMP-2 ${ }^{-I-}$ Mice}

To clarify the role of MMP-2 in interstitial fibrogenesis, MMP-2 $2^{-l-}$ mice were subjected to UUO. The kidneys of MMP- $2^{-1-}$ mice showed minimal interstitial fibrosis with mild deposition of type III and type I collagens (Figures 1 and 2). Gelatin zymography and real-time PCR study confirmed the lack of production and activity of MMP-2 during the experiment (Figures 3 and 4). MMP-9 activity increased by day 3 , similar to MMP- $2^{+/+}$mice. From days 3 to 14 post-UUO, MMP-9 activity gradually increased, reaching a peak level at day 14 post-UUO. Comparison of MMP- $2^{-/-}$ and $\mathrm{MMP}-2^{+/+}$mice showed similar production and activity of MMP-9 at both baseline and post-UUO, and no upregulation of MMP-9 in MMP-2 $2^{-1-}$ mice before and after UUO, compared with MMP- $2^{+/+}$mice. The in situ zymography demonstrated that gelatinase activity was also lower in MMP $-2^{-/-}$mice than MMP- $2^{+/+}$mice (Figure 5), which may be associated with MMP-2 deficiency. During the development of fibrosis in MMP-2 ${ }^{-1-}$ mice, the expression levels of S100A4, vimentin, HSP-47, and $\alpha$ SMA + cells were less than the respective levels in MMP- $2^{+1+}$ mice (Figures $6-8), F 4 / 80+$ macrophage infiltration was also inhibited in MMP- $2^{-1-}$ mice. With regard to the EMT-related molecules, the upregulation of ILK, TGF- $\beta / \mathrm{Smad}$, and $\mathrm{Wnt} / \beta$-catenin signaling, and Snail was inhibited in MMP- $2^{-1-}$ mice at day 14 (Figure 9). Electron microscopy in MMP- $2^{-1-}$ mice at day 14 showed loss of basolateral infolding and surface microvilli in tubular epithelial cells, the lack of spindle-shape epithelial cells and no clear changes in TBM (Figure 10). Thus, the lack of MMP-2 reduced renal interstitial fibrosis post-UUO possibly through the suppression of tubular cell EMT, inhibition of myofibroblast accumulation, and suppression of macrophage infiltration.

\section{UUO in MMP-2 ${ }^{+1+}$ Mice Treated with Minocycline}

Finally, we examined the effects of MMP inhibitor on renal fibrosis by injecting minocycline in UUO-MMP- $2^{+/+}$mice. Minocycline reduced interstitial fibrosis at 14 days postUUO, as determined by the Masson stain + areas and the deposition of type III and type I collagens (Figures 1 and 2). Minocycline inhibited the activities and the production of MMP-2 and MMP-9 (Figures 3 and 4). In addition, minocycline suppressed gelatinase activity in vivo (Figure 5). Minocycline reduced the expression of S100A4, vimentin, $\alpha$ SMA, and HSP-47 in the kidney at day 14 post-UUO (Figures 6-8). F4/80 + macrophage infiltration was also inhibited by minocycline. With regard to EMT-related molecules, minocycline suppressed the upregulated expression of ILK, TGF- $\beta /$ Smad, and Wnt $/ \beta$-catenin signaling, and Snail at day 14 (Figure 9). Electron microscopic examination of MMP- $2^{+/+}$mice treated with minocycline at day 14 showed minimal changes in tubular epithelial cells and TBM. In damaged tubules, basolateral in folding and surface microvilli of tubular epithelial cells were well preserved with mild changes in TBM (Figure 10). These results suggest that inhibition of MMP-2 and MMP-9 reduces interstitial fibrogenesis by possibly protecting EMT promotion and macrophage infiltration.

Importantly, the severity of the renal interstitial fibrosis including the deposition of type III and type I collagens, and the expression of S100A4, vimentin, $\alpha \mathrm{SMA}$, and HSP-47, was less prominent in $\mathrm{MMP}-2^{-/-}$mice and $\mathrm{MMP}-2^{+/+}$ mice treated with minocycline than those in MMP- $2^{+1+}$ mice. Furthermore, it was less prominent in MMP- $2^{+1+}$ mice treated with minocycline than in MMP- $2^{-/-}$mice. These
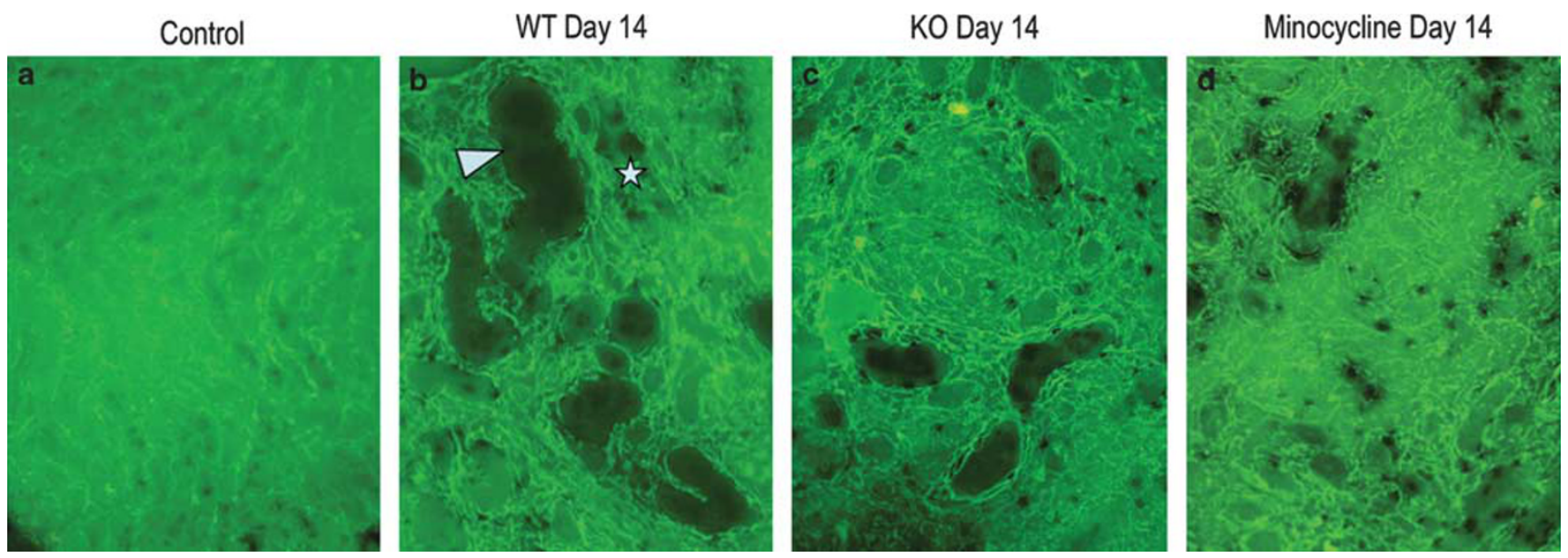

Figure 5 MMP-2 and MMP-9 activities examined by in situ zymography in the kidneys of sham-operated control (a: Control) and at day 14 post-unilateral ureteral obstruction (UUO) in MMP-2 $2^{+/+}$mice (b: WT Day 14), MMP-2 ${ }^{-1-}$ mice (c: KO Day 14), and MMP-2 $2^{+/+}$mice treated with minocycline (d: Minocycline Day 14) (a-d: $\times 200)$. Note the gelatinase activity at day 14 mainly in dilated (arrowhead in panel b) and atrophic (asterisk in panel b) damaged tubules. However, the gelatinase activity was suppressed in $\mathrm{MMP}-2^{-/-}$mice and MMP- $2^{+/+}$mice treated with minocycline at day 14 post-UUO. 

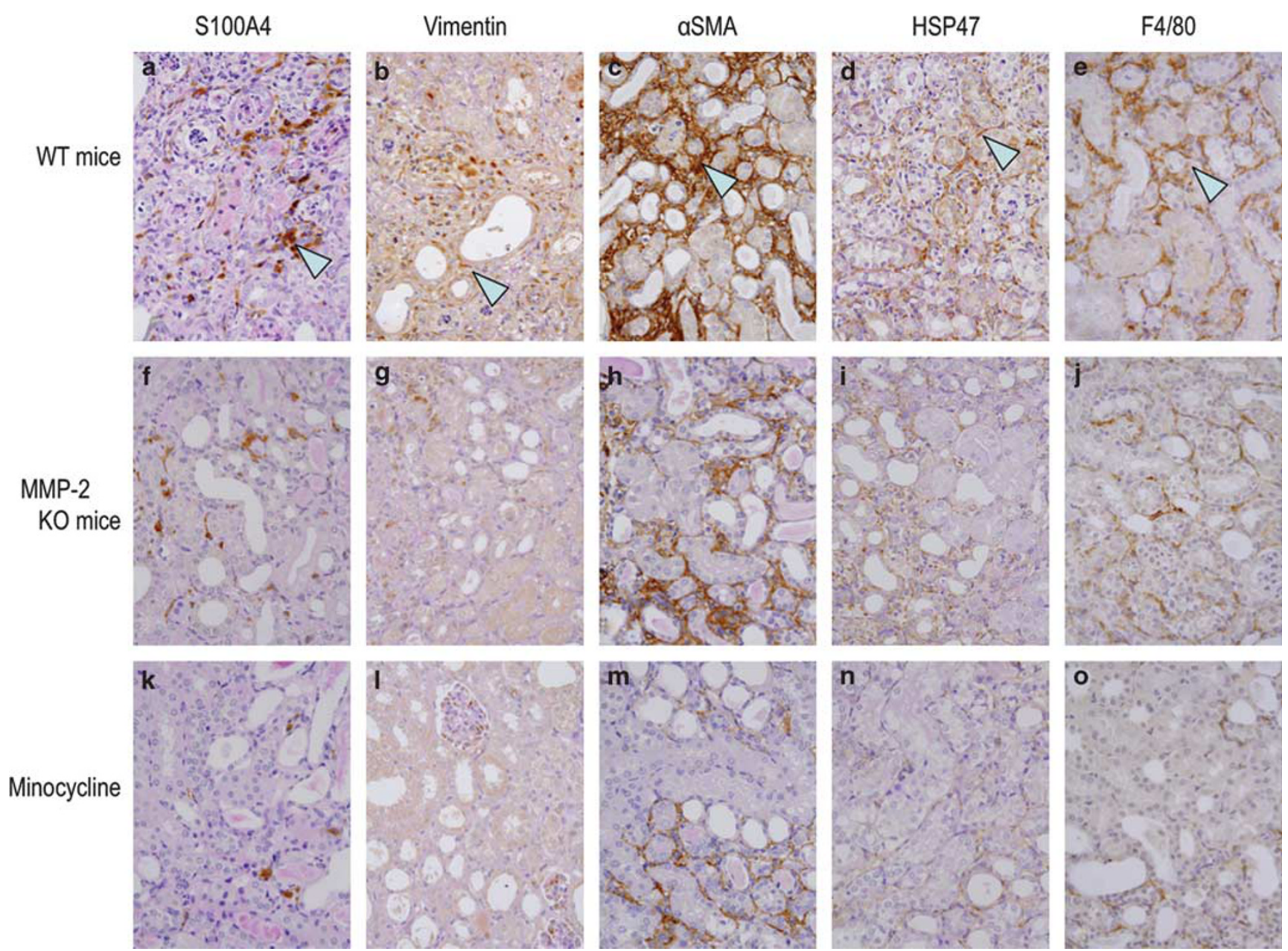

Figure 6 The expression of mesenchymal marker proteins, including S100A4 (a, $\mathbf{f}, \mathbf{k})$, vimentin $(\mathbf{b}, \mathbf{g}, \mathbf{l})$, and $\alpha$-smooth muscle actin $(\alpha$ SMA) (c, $\mathbf{h}, \mathbf{m})$, marker of collagen synthesis heat shock protein-47 (HSP-47), which is known as the collagen-specific molecular chaperone (d, i, $\mathbf{n}$ ), and macrophage infiltration (F4/ 80) $(\mathbf{e}, \mathbf{j}, \mathbf{o})$, at day 14 post-unilateral ureteral obstruction (UUO) in MMP- $2^{+/+}$mice (a-e: WT mice), MMP-2 ${ }^{-/-}$mice (f-j: MMP-2 KO mice), and MMP-2 $2^{+/+}$ mice treated with minocycline (k-o: Minocycline) $(\mathbf{a}-\mathbf{o}, \times 400)$. In MMP- $2^{+/+}$mice at day 14 post-UUO, the expression of S100A4, vimentin, $\alpha$ SMA, and HSP-47 was evident in damaged tubular epithelial cells and interstitial cells (arrowhead in panels a-d). Infiltration of macrophages was noted around tubules (arrowhead in panel e). However, they were less prominent in MMP-2 ${ }^{-1-}$ mice and MMP- $2^{+/+}$mice treated with minocycline than those in $\mathrm{MMP}-2^{+/+}$mice.

results show that although the lack of MMP-2 and inhibition of MMP-2 and MMP-9 meditated the suppression of tubular cell EMT findings and reduction of macrophage infiltration, these actions were strongly modulated by inhibition of both MMP-2 and MMP-9 compared with inhibition of MMP-2 alone.

\section{DISCUSSION}

MMP activity is assumed to be necessary for the dissolution of ECM and proteolytic removal of interstitial fibrosis in renal diseases. However, this study demonstrated the involvement of the expression of MMPs, especially MMP-2, probably in the promotion of EMT in tubular epithelial cells through the degradation of TBM, which could be associated with the progression of interstitial fibrosis. The lack of MMP-2 and inhibition of MMP-2 and MMP-9 suppressed tubular cell EMT findings, and protected against the progression of renal interstitial fibrosis. The results provide the background for the design of new pharmacologic agents that can reduce renal fibrosis in chronic renal diseases.

In recent years, EMT has attracted interest in the field of chronic renal diseases. ${ }^{1-12}$ Damaged tubular epithelial cells have mesenchymal phenotype through EMT, and migrate into interstitium. ${ }^{7-12}$ Indeed, one recent study using a gGTLacZ transgenic mice, which allows the indisputable identification of cells derived from proximal tubular epithelium in the kidney, reported that more than one-third of renal interstitial fibroblasts were derived from renal tubular epithelium through EMT. ${ }^{28}$ MMP-2 and MMP-9, which mediate TBM degradation, are considered a critical event in renal EMT. ${ }^{29,30}$ MMP-2 appears both necessary and sufficient to induce tubular EMT. ${ }^{19,21}$ Similarly, a recent study using transgenic mice with targeted expression of active MMP-2 in the proximal tubular epithelial cells has demonstrated 

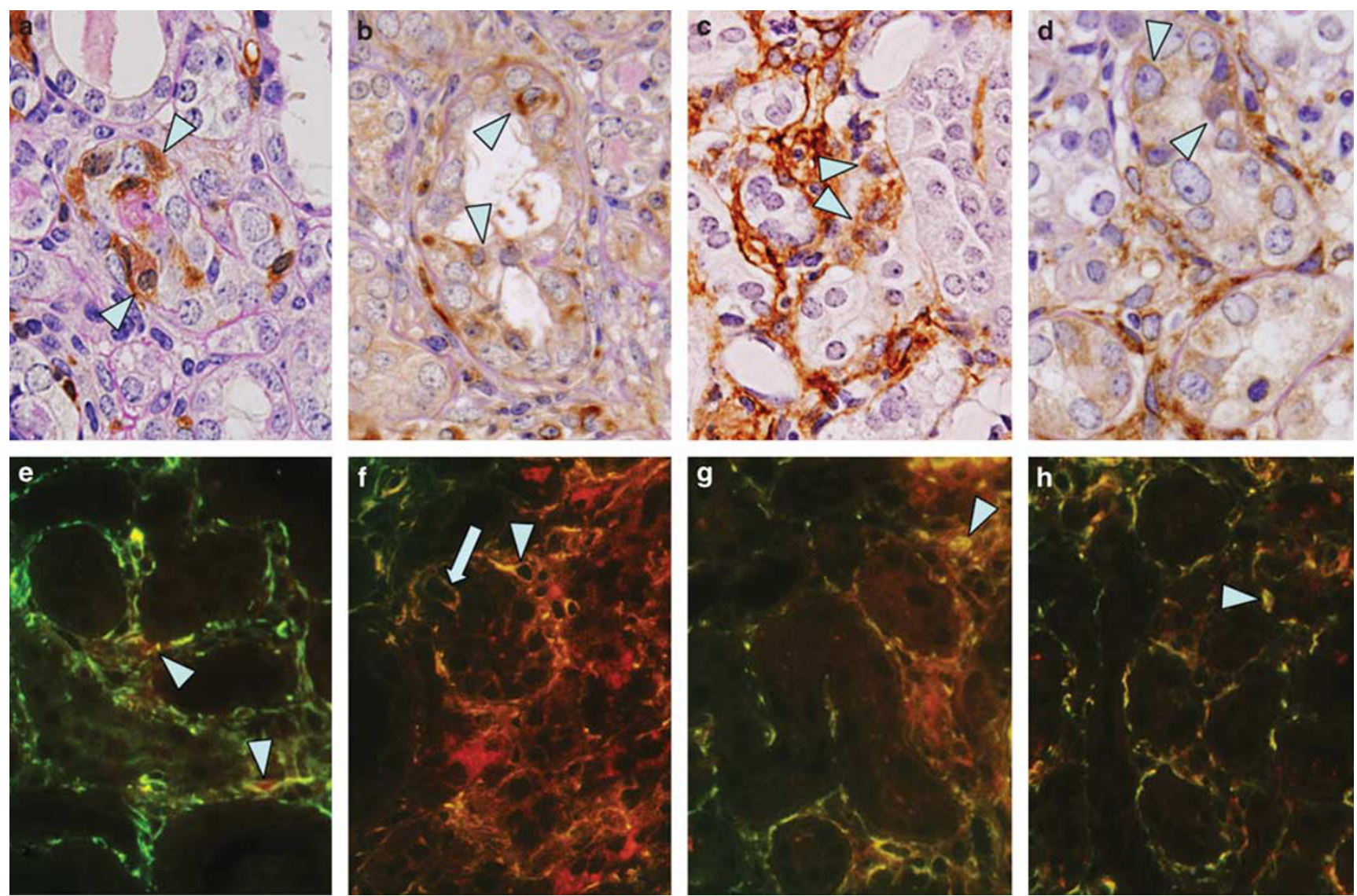

Figure 7 The expression of S100A4 $(\mathbf{a}, \times 800)$, vimentin $(\mathbf{b}, \times 800), \alpha$-smooth muscle actin $(\alpha \mathrm{SMA})(\mathbf{c}, \times 800)$, and heat shock protein-47 (HSP-47, $\times 800)$ (d) in renal tubules at day 14 post-unilateral ureteral obstruction (UUO), and double immunostaining with S100A4 (green) and $\alpha$ SMA (red) before UUO (e) and at day 14 post-UUO in MMP-2 $2^{+/+}$mice $(\mathbf{f}), \mathrm{MMP}_{-} 2^{-/-}$mice $(\mathbf{g})$, and MMP- $2^{+/+}$mice treated with minocycline $(\mathbf{h})(\mathbf{e}-\mathbf{h}, \times 600)$. S100A4,+ vimentin + , $\alpha \mathrm{SMA}+$, or HSP-47 + cells were present in damaged renal tubules (arrowhead in panels a-d). In addition, both S100A4+ (green) and $\alpha \mathrm{SMA}+($ red) myofibroblastic cells were present around tubules (arrowhead in panels $\mathbf{e}-\mathbf{h}$ ), and these cells were also present in renal tubules (arrow in panel $\mathbf{f}$ ) at day 14 post-UUO in MMP-2 ${ }^{+/+}$mice.

directly that active MMP-2 alone is sufficient to drive the full spectrum of EMT. ${ }^{21}$ Therefore, in addition to the pathogenic role of MMP-2 activity in the migration of tubular epithelial cells with mesenchymal phenotype into the interstitium accompanied by the destruction of TBM, recent studies have also indicated that MMP-2 can trigger EMT, probably through the disruption of TBM integrity.

Several current studies also indicate that EMT is unlikely to occur in vivo. ${ }^{13-15}$ The comprehensive lineage tracing studies demonstrate that there is no evidence for EMT as a source of myofibroblasts in vivo in the kidney. ${ }^{13-15}$ Rather, vascular pericytes or local interstitial fibroblasts are the source of fibrosis-generating myofibroblasts. ${ }^{14,15}$ Our previous study of chronic rejection in renal transplantation model demonstrated that interstitial $\alpha \mathrm{SMA}+$ myofibroblasts initially occurred around peritubular capillaries and progressed into interstitium during the development of renal fibrosis. ${ }^{31}$ Unfortunately, we did not analyze the pericytes around peritubular capillaries or local interstitial fibroblasts in detail in this study, because MMP-2 expressed mainly damaged renal tubular cells. In this study, we have considered that EMT may be involved in the fibrogenesis in the mice UUO model, although this is not the only mechanism, as (1) damaged tubular epithelial cells expressed mesenchymal and collagen production associated proteins, such as S100A4, vimentin, $\alpha \mathrm{SMA}$, and HSP47; (2) both $\alpha \mathrm{SMA}+$ and S100A4 + myofibloblasts were present within renal tubules, although the number of these cells were only a few; (3) our electron microscopic findings indicated the spindle cells were present between renal tubules and interstitium through ruptured TBM; and (4) central mediator signalings of renal tubular cell EMT, such as ILK, TGF- $\beta /$ Smad, Wnt $/ \beta$-catenin, and Snail, were upregulated in the development of renal fibrosis.

Tubular epithelial cells are tightly connected with TBM via cell-ECM adhesion molecules such as integrin, and they are regulated microenvironment for their function. ${ }^{32-36}$ In the cell-ECM interactions, integrin-mediated signals from the ECM are transduced by ILK, a cytoplasmic serine-threonine kinase, and the activated ILK upregulate the both $\beta$-catenin 

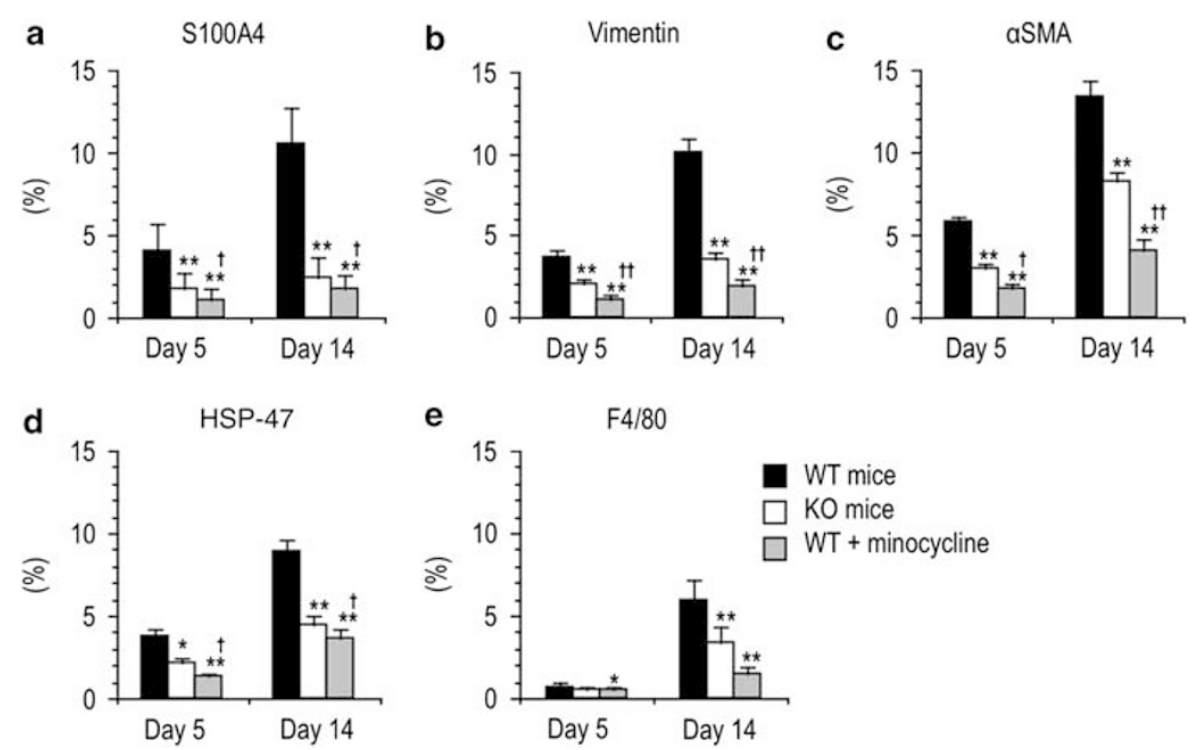

Figure 8 Quantitative analysis of the expression of S100A4 (a), vimentin (b), $\alpha$-smooth muscle actin ( $\alpha$ SMA) (c), heat shock protein-47 (HSP-47) (d), and F4/80 (e) in MMP-2 $2^{+1+}$ mice (WT mice), MMP- $2^{-1-}$ mice (KO mice) and MMP- $2^{+/+}$mice treated with minocycline (WT + minocycline) at day 5 and day 14 post-UUO. Data are mean \pm s.d. of $n=5$ mice. ${ }^{*} P<0.05$ and ${ }^{* *} P<0.001$ are compared with MMP- $2^{+/+}$mice. ${ }^{\dagger} P<0.05$ and ${ }^{\dagger \dagger} P<0.001$ are on MMP-2 ${ }^{+1+}$ mice treated with minocycline, which is compared with $\mathrm{MMP}^{-2^{-1}}$ mice.
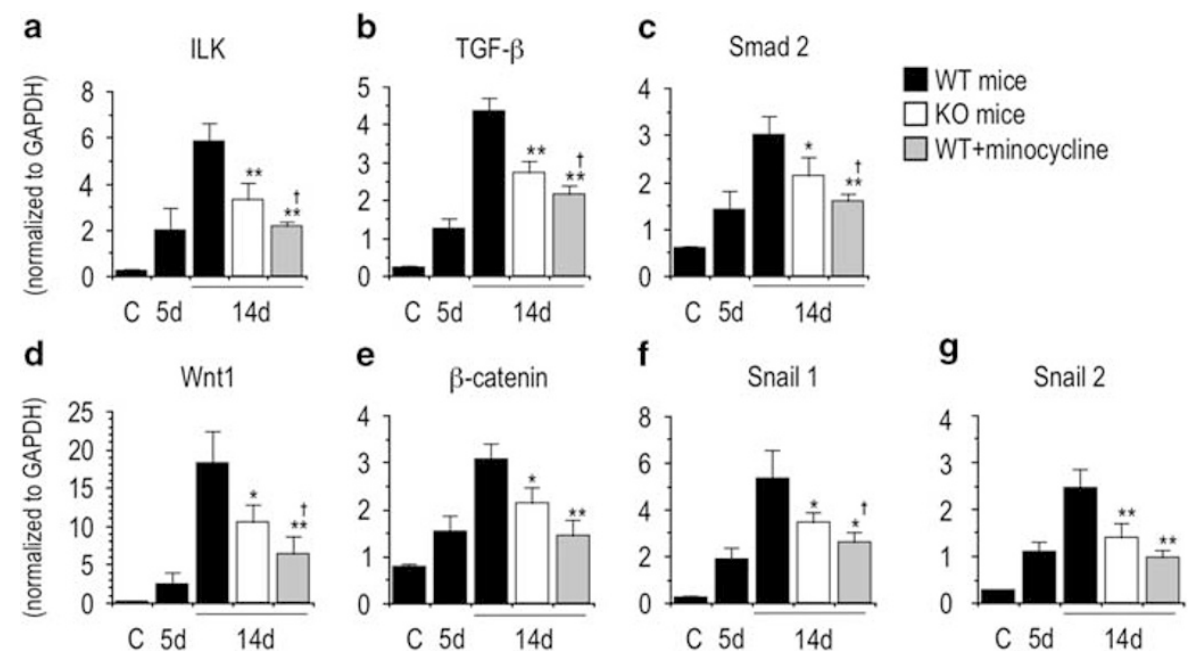

Figure 9 The expression of epithelial-to-mesenchymal transition (EMT)-related molecules. mRNA levels of integrin-linked kinase (ILK) (a), transforming growth factor- $\beta 1$ (TGF- $\beta 1)(\mathbf{b})$, Smad 2 (c), Wnt1 (d), $\beta$-catenin (e), Snail 1 (f), and Snail 2 (g) were upregulated at day 5 (5d) and day 14 (14d) post-unilateral ureteral obstruction (UUO) in MMP-2 ${ }^{+/+}$mice (WT mice) from before UUO control (C). The EMT-related molecules significantly inhibited at day 14 post-UUO in MMP-2 $2^{-1-}$ mice (KO mice) and MMP-2 ${ }^{+/+}$mice treated with minocycline (WT + minocycline). Data are mean \pm s.d. of $n=5$ mice. ${ }^{\star P}<0.05$ and ${ }^{*} P<0.001$ are compared with MMP-2 ${ }^{+/+}$mice. ${ }^{\dagger} P<0.05$ is on MMP-2 ${ }^{+/+}$mice treated with minocycline, which is compared with MMP-2 ${ }^{-/-}$mice.

and Snail that is known as molecules involving EMT. ${ }^{37-39}$ MMPs are involved in the regulation of cell-ECM interactions. ${ }^{40}$ In addition, MMP-2 facilitates the production of active TGF- $\beta 1$ protein, which is known as a key factor for EMT promotion. ${ }^{19}$ In this study, UUO induced the production of ILK and $\beta$-catenin and upregulation of Snail. In addition, TGF- $\beta 1 /$ Smad and $\mathrm{Wnt} / \beta$-catenin signaling molecules, which are known as central mediator signaling of renal tubular cell EMT, were also upregulated during the process of renal fibrosis.

To examine the possible use of MMP inhibitors in the treatment of renal fibrosis, UUO was induced in MMP- $2^{-1-}$ mice and in MMP- $2^{+1+}$ mice treated with minocycline. Minocycline has a role in the inhibition of MMP-2 and MMP-9. ${ }^{41}$ In this study, minocycline could inhibit activities and production of MMP-2 and MMP-9. The kidneys of 

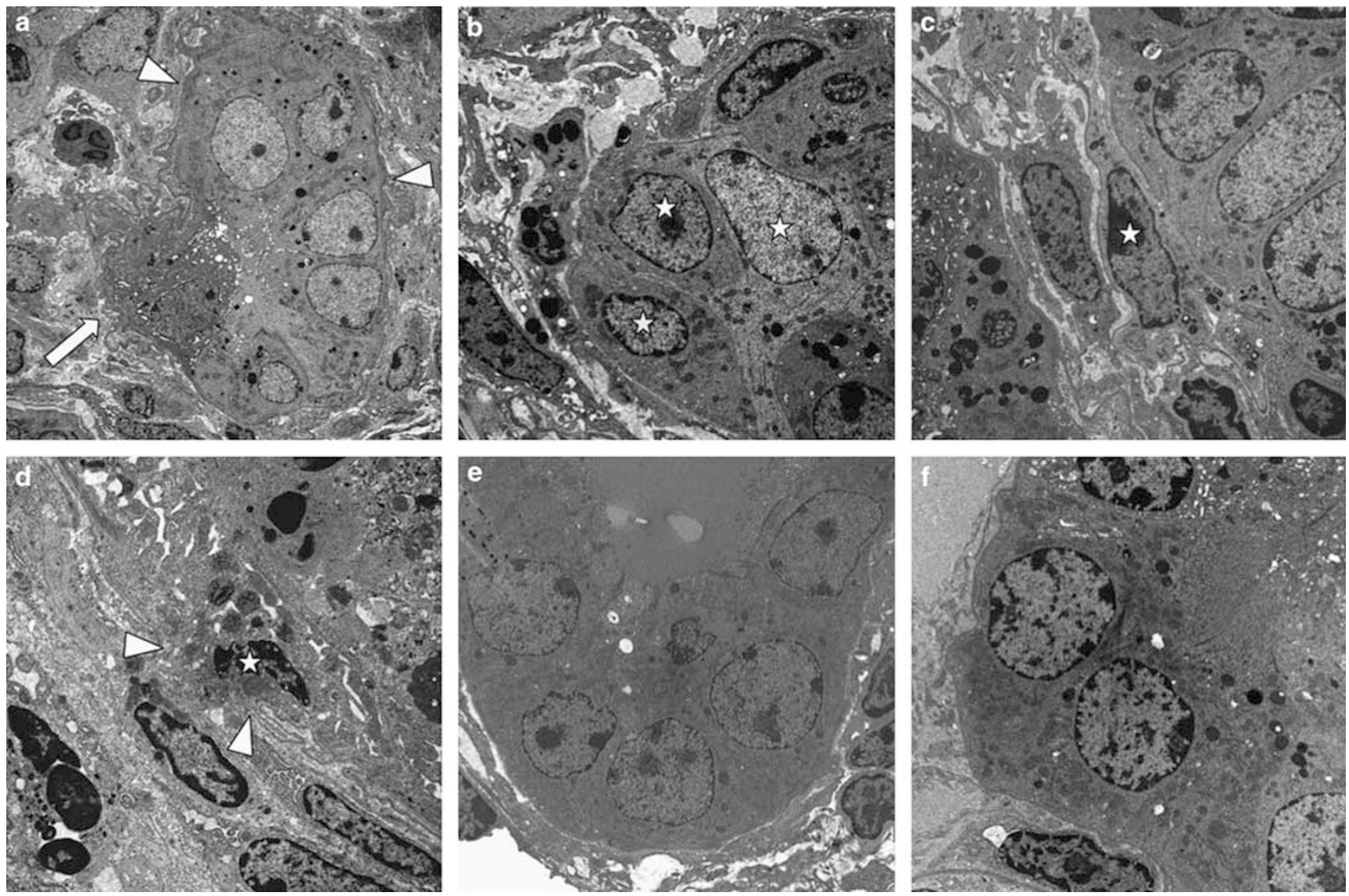

Figure 10 Electron microscopic findings at day 14 post-unilateral ureteral obstruction (UUO) in MMP-2 $2^{+/+}$mice (a-d), MMP-2 $2^{-/-}$mice (e), and MMP-2 $2^{+/+}$ mice treated with minocycline (f). At day 14 post-UUO in MMP-2 ${ }^{+1+}$ mice, atrophic tubules and damaged epithelial cells were present with loss of basolateral in folding, loss of surface microvilli, and presence of filamentous actin bundles (arrowhead in panel $\mathbf{a}, \times 2000$ ). Tubular basement membrane (TBM) was redundant and showed irregular thickening and tortuous profile (arrow in panel a). In some atrophic tubules, spindle-shape tubular epithelial cells (asterisk in panel $\mathbf{b}, \times 8000$ ) were noted with ill-defined tight junctions. Fibroblast-like mesenchymal cells (asterisk in panel $\mathbf{c}, \times 15000$ ) were present in the basal area between damaged epithelial cells and TBM. The EMT foci were characterized by TBM disruption (between arrowheads in panel $\mathbf{d}, \times 12000$ ) and interstitial invasion of spindle-shape epithelial cells (asterisk in panel $\mathbf{d}$ ) with accumulation of fibroblastic cells around TBM. In MMP $-2^{-1-}$ mice $(\mathbf{e}, \times 4000)$, epithelial cells lost their basolateral in folding and surface microvilli, but preserved contact with TBM. In MMP-2 $2^{+/+}$ mice treated with minocycline $(\mathbf{f}, \times 6000)$, tubular epithelial cells showed well-preserved structure and contact with TBM.

MMP- $2^{-1-}$ and MMP- $2^{+1+}$ mice treated with minocycline showed reduced EMT findings in tubular epithelial cells and preservation of renal fibrosis after UUO. Importantly, the ameliorative effects of minocycline on the severity of renal fibrosis were more pronounced than those of MMP- $2^{-1-}$ mice. These results show that MMP-2 has an important role in the promotion of tubular cell EMT findings as well as the development of renal fibrosis. Furthermore, inhibition of MMP-2 and MMP-9 may be helpful in the restoration or maintenance of an epithelial phenotype, suppression of EMT findings, and inhibition of interstitial fibrosis.

Although inhibition of MMP-2 and MMP-9 mitigates tubular cell EMT findings, we have to consider also the possible role of the inflammatory cascade in the development of interstitial fibrosis after UUO. Macrophage MMP-9 mediates EMT in renal tubular cells. ${ }^{42}$ MMP activity is usually linked to inflammation through interactions with and activation of several cytokines, chemokines and other pericellular and cell surface substrates. ${ }^{43-45}$ Minocycline has broad anti-inflammatory properties, in addition to its action as an MMP inhibitor. ${ }^{41}$ In this study, many F4/80+ macrophages infiltrated around damaged tubules following UUO, which were suppressed in MMP- $2^{-1-}$ mice or following treatment with minocycline. Thus, MMP-2 alone or MMP-2 and MMP-9 seem to enhance renal inflammation and contribute to the development of renal fibrosis.

In summary, the tubular epithelium, because of its unique mesenchymal origin, seems capable of acquiring mesenchymal phenotype in disease through EMT, and contributes to renal fibrosis in chronic renal diseases. This study demonstrated an important role for MMP-2 in the pathogenesis of renal fibrosis, that is, the probably induction and promotion of EMT findings. Induction of MMP-2 and targeted proteolysis of the TBM provides a unifying mechanism that 
integrates the multiple pathophysiological processes that induce epithelial cell injury. Strategies aimed at inhibition of MMP-2 alone or MMP-2 and MMP-9, especially in the early stages of fibrosis, could reduce progressive fibrosis in chronic kidney diseases.

\section{ACKNOWLEDGEMENTS}

We express special thanks to Ms Kyoko Wakamatsu and Ms Arimi Ishikawa for the expert technical assistance. We are also grateful to Drs Akiko Mii and Emiko Fujita, Department of Internal Medicine (Division of Neurology, Nephrology and Rheumatology), Nippon Medical School, for their advice.

\section{DISCLOSURE/CONFLICT OF INTEREST}

The authors declare no conflict of interest.

1. Hewitson TD. Renal tubulointerstitial fibrosis: common but never simple. Am J Physiol Renal Physiol 2009;296:F1239-F1244.

2. Zeisberg M, Neilson EG. Mechanisms of tubulointerstitial fibrosis. J Am Soc Nephrol 2010;21:1819-1834.

3. Boor P, Ostendorf $T$, Floege J. Renal fibrosis: novel insights into mechanisms and therapeutic targets. Nat Rev Nephrol 2010;6:643-656.

4. Zeisberg $M$, Duffield JS. Resolved: EMT produces fibroblasts in the kidney. J Am Soc Nephrol 2010;21:1247-1253.

5. Quaggin SE, Kapus A. Scar wars: mapping the fate of epithelialmesenchymal-myofibroblast transition. Kidney Int 2011;80:41-50.

6. Meran S, Steadman R. Fibroblasts and myofibroblasts in renal fibrosis. Int J Exp Pathol 2011;92:158-167.

7. Burns WC, Kantharidis $P$, Thomas MC. The role of tubular epithelialmesenchymal transition in progressive kidney disease. Cells Tissues Organs 2007;185:222-231.

8. Bani-Hani AH, Campbell MT, Meldrum DR, et al. Cytokines in epithelialmesenchymal transition: a new insight into obstructive nephropathy. J Urol 2008;180:461-468.

9. Guarino M, Tosoni A, Nebuloni M. Direct contribution of epithelium to organ fibrosis: epithelial-mesenchymal transition. Hum Pathol 2009;40:1365-1376.

10. Zeisberg $M$, Neilson EG. Biomarkers for epithelial-mesenchymal transitions. J Clin Invest 2009;119:1429-1437.

11. Liu Y. New insights into epithelial-mesenchymal transition in kidney fibrosis. J Am Soc Nephrol 2010;21:212-222.

12. Fragiadaki $M$, Mason RM. Epithelial-mesenchymal transition in renal fibrosis - evidence for and against. Int J Exp Pathol 2011;92:143-150.

13. Li L, Zepeda-Orozco D, Black R, et al. Autophagy is a component of epithelial cell fate in obstructive uropathy. Am J Pathol 2010;176:1767-1778.

14. Humphreys $\mathrm{BD}$, Lin $\mathrm{SL}$, Kobayashi $\mathrm{A}$, et al. Fate tracing reveals the pericyte and not epithelial origin of myofibroblasts in kidney fibrosis. Am J Pathol 2010;176:85-97.

15. Koesters $R$, Kaissling $B$, Lehir $M$, et al. Tubular overexpression of transforming growth factor beta 1 induces autophagy and fibrosis but not mesenchymal transition of renal epithelial cells. Am J Pathol 2010;177:632-643.

16. Nagase $H$, Visse $R$, Murphy $G$. Structure and function of matrix metalloproteinases and TIMPs. Cardiovasc Res 2006;69:562-573.

17. Catania JM, Chen G, Parrish AR. Role of matrix metalloproteinases in renal pathophysiologies. Am J Physiol Renal Physiol 2007;292: F905-F911.

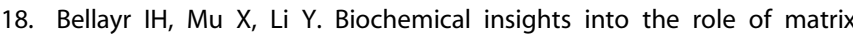
metalloproteinases in regeneration: challenges and recent developments. Future Med Chem 2009;1:1095-1111.

19. Cheng S, Lovett DH. Gelatinase A (MMP-2) is necessary and sufficient for renal tubular cell epithelial-mesenchymal transformation. Am J Pathol 2003;162:1937-1949.
20. Liu Y. Epithelial to mesenchymal transition in renal fibrogenesis: pathologic significance, molecular mechanism, and therapeutic intervention. J Am Soc Nephrol 2004;15:1-12.

21. Cheng S, Pollock AS, Mahimkar R, et al. Matrix metalloproteinase 2 and basement membrane integrity: a unifying mechanism for progressive renal injury. FASEB J 2006;20:1898-1900.

22. Wang $X$, Zhou $Y$, Tan $R$, et al. Mice lacking the matrix metalloproteinase- 9 gene reduce renal interstitial fibrosis in obstructive nephropathy. Am J Physiol Renal Physiol 2010;299:F973-F982.

23. Klahr S, Morrissey J. Obstructive nephropathy and renal fibrosis. Am J Physiol Renal Physiol 2002;283:F861-F875.

24. Chevalier RL, Forbes MS, Thornhill BA. Ureteral obstruction as a model of renal interstitial fibrosis and obstructive nephropathy. Kidney Int 2009;75:1145-1152.

25. Itoh $\mathrm{T}$, Ikeda $\mathrm{T}$, Gomi $\mathrm{H}$, et al. Unaltered secretion of beta-amyloid precursor protein in gelatinase A (matrix metalloproteinase 2)deficient mice. J Biol Chem 1997;272:22389-22392.

26. Kunugi $\mathrm{S}$, Shimizu A, Kuwahara $\mathrm{N}$, et al. Inhibition of matrix metalloproteinases reduces ischemia-reperfusion acute kidney injury. Lab Invest 2011;91:170-180.

27. Kunugi S, Fukuda $Y$, Ishizaki $M$, et al. Role of MMP-2 in alveolar epithelial cell repair after bleomycin administration in rabbits. Lab Invest 2001;81:1309-1318.

28. Iwano M, Plieth D, Danoff TM, et al. Evidence that fibroblasts derive from epithelium during tissue fibrosis. J Clin Invest 2002;110:341-350.

29. Zeisberg M, Maeshima Y, Mosterman B, et al. Renal fibrosis. Extracellular matrix microenvironment regulates migratory behavior of activated tubular epithelial cells. Am J Pathol 2002;160:2001-2008.

30. Zeisberg $M$, Bonner $G$, Maeshima $Y$, et al. Renal fibrosis: collagen composition and assembly regulates epithelial-mesenchymal transdifferentiation. Am J Pathol 2001;159:1313-1321.

31. Shimizu A, Yamada K, Sachs DH, et al. Persistent rejection of peritubular capillaries and tubules is associated with progressive interstitial fibrosis. Kidney Int 2002;61:1867-1879.

32. Nony PA, Schnellmann RG. Interactions between collagen IV and collagen-binding integrins in renal cell repair after sublethal injury. Mol Pharmacol 2001;60:1226-1234.

33. Berrier AL, Yamada KM. Cell-matrix adhesion. J Cell Physio 2007;213:565-573.

34. Lelongt $B$, Ronco P. Role of extracellular matrix in kidney development and repair. Pediatr Nephrol 2003;18:731-742.

35. Daley WP, Peters SB, Larsen M. Extracellular matrix dynamics in development and regenerative medicine. J Cell Sci 2008;121:255-264.

36. Gjorevski N, Nelson CM. Bidirectional extracellular matrix signaling during tissue morphogenesis. Cytokine Growth Factor Rev 2009:20:459-465.

37. Wu C, Dedhar S. Integrin-linked kinase (ILK) and its interactors: a new paradigm for the coupling of extracellular matrix to actin cytoskeleton and signaling complexes. J Cell Biol 2001;155:505-510.

38. Li Y, Yang J, Dai C, et al. Role for integrin-linked kinase in mediating tubular epithelial to mesenchymal transition and renal interstitial fibrogenesis. J Clin Invest 2003;112:503-516.

39. Li $Y$, Tan $X$, Dai $C$, et al. Inhibition of integrin-linked kinase attenuates renal interstitial fibrosis. J Am Soc Nephrol 2009;20:1907-1918.

40. Ronco P, Chatziantoniou C. Matrix metalloproteinases and matrix receptors in progression and reversal of kidney disease: therapeutic perspectives. Kidney Int 2008;74:873-878.

41. Elewa HF, Hilali H, Hess DC, et al. Minocycline for short-term neuroprotection. Pharmacotherapy 2006;26:515-521.

42. Tan TK, Zheng G, Hsu TT, et al. Macrophage matrix metalloproteinase-9 mediates epithelial-mesenchymal transition in vitro in murine renal tubular cells. Am J Pathol 2010;176:1256-1270.

43. Manicone AM, McGuire JK. Matrix metalloproteinases as modulators of inflammation. Semin Cell Dev Biol 2008;19:34-41.

44. Gill SE, Parks WC. Metalloproteinases and their inhibitors: regulators of wound healing. Int J Biochem Cell Biol 2008;40:1334-1347.

45. Korpos $\mathrm{E}, \mathrm{Wu} \mathrm{C}$, Sorokin L. Multiple roles of the extracellular matrix in inflammation. Curr Pharm Des 2009;15:1349-1357. 